\title{
Transcriptomic and Metabolomic Analyses Provide Insights into the Growth and Development Advantages of Triploid Apostichopus japonicus
}

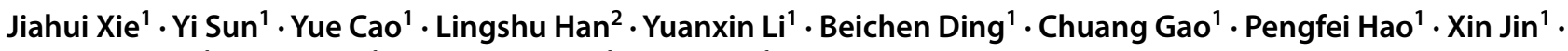 \\ Yaqing Chang ${ }^{1} \cdot$ Jian Song ${ }^{1} \cdot$ Donghong Yin ${ }^{1} \cdot$ Jun Ding $^{1}$
}

Received: 21 October 2021 / Accepted: 17 January 2022 / Published online: 5 February 2022

(c) The Author(s) 2022

\begin{abstract}
Polyploid breeding is widely used in aquaculture as an important area of new research. We have previously grown Apostichopus japonicus triploids with a growth advantage. The body length, body weight, and aestivation time of triploid and diploid A. japonicus were measured in this study, and the transcriptome and metabolome were used to examine the growth advantage of triploids A. japonicus. The results showed that the proportion of triploid A. japonicus with a body length of 6-12 cm and 12-18 cm was significantly higher than that of diploid A. japonicus, and triploid A. japonicus had a shorter aestivation time (39 days) than diploid (63 days). We discovered 3296 differentially expressed genes (DEGs); 13 DEGs (for example, cyclin-dependent kinase 2) related to growth advantage, immune regulation, and energy storage were screened as potential candidates. According to Gene Ontology (GO) enrichment analysis, DEGs were significantly enriched in the cytoplasm (cellular component), ATP binding process (molecular function), oxidation-reduction process (biological process), and other pathways. According to the Kyoto Encyclopedia of Genes and Genomes (KEGG) enrichment data, DEGs were significantly enriched in ribosome production and other areas. We discovered 414 significant differential metabolites (SDMs), with 11 important SDMs (for example, nocodazole) linked to a growth advantage. SDMs are significantly enriched in metabolic pathways, as well as other pathways, according to the KEGG enrichment results. According to a combined transcriptome and metabolome analysis, 6 DEGs have regulatory relationships with 11 SDMs, which act on 11 metabolic pathways together. Our results further enrich the biological data of triploid A. japonicus and provide useful resources for genetic improvement of this species.
\end{abstract}

Keywords Triploid Apostichopus japonicus $\cdot$ Transcriptome $\cdot$ Metabolome $\cdot$ Growth $\cdot$ Aestivation

\section{Introduction}

Polyploidy, which was first proposed by Winkler (1916), refers to the occurrence of three or more genomes in each somatic cell (Joana et al. 2018). Most polyploids naturally

Jiahui Xie, Yi Sun contributed equally to this work.

Jun Ding

dingjun1119@dlou.edu.cn

1 Key Laboratory of Mariculture \& Stock Enhancement in, Ministry of Agriculture and Rural Affairs, North China's Sea, Dalian Ocean University, Dalian, Liaoning, People's Republic of China 116023

2 Ningbo University, Ningbo, Zhejiang, People's Republic of China 315211 exist in fish but, at present, artificial polyploidy is frequently used. Polyploid breeding first appeared in the artificial induction of triploid carp by Makino and Ojima (Ren et al. 2018). Since then, polyploid breeding of aquatic animals has been widely used in fish and shellfish. Polyploid breeding has good market value in shellfish. Guo invented the production method for tetraploid mollusks (oysters, scallops, clams, mussels, and abalones) in 1995, and proposed the method of producing triploid by mating tetraploid and diploid (Guo and Allen 1994; Guo et al. 1996). Compared with diploid oysters, triploid oysters have the advantages of large size, fast growth (Guo et al. 2009; Guo 2021), and high nutritional value (Qin et al. 2018). At present, polyploid breeding has been applied or studied in more than 40 types of fish and more than 20 types of economic shellfish and crustaceans (Song et al. 2004). 
Apostichopus japonicus (Echinodermata: Holothuroidea: Aspidochirotida: Stichopodidae) is an important economic echinoderm, and is a species known to be of the best quality among the more than 20 types of edible sea cucumbers. However there have been few studies on the polyploid breeding of echinoderms, except that Chang successfully induced triploid and tetraploid A. japonicus using cytochalasin B and 6-dimethylaminopurine (6-DMAP), and examined the inducing drug concentration, treatment time, treatment start time, and the survival rate of larvae (Chang and Xiang 2002). Ding then proposed the method of inducing triploid A. japonicus by hydrostatic pressure (Ding et al. 2007). Han et al (2021) used transcriptome and methylation to study the role of methylation changes of different genes in triploid $A$. japonicus. However, the molecular mechanism of controlling the dominant traits such as fast growth and short aestivation time of triploid A. japonicus is not clear. Therefore, we expect to determine the significantly different genes, metabolites, and metabolic pathways related to triploid $A$. japonicus which participate in growth immunity, and understand the relationship between different genes and different metabolites.

In recent years, exploring the growth and development (Sun et al. 2017; Xing et al. 2021), immune function (Li et al. 2018; Shi et al. 2020), and phylogeny (Zhao et al. 2020; Carmona et al. 2017) of aquaculture animals using various omic techniques have gradually become a research hotspot (Chen et al. 2020). The combined analysis of transcriptome and metabolome is widely used to reveal the molecular mechanism in organisms (Wang et al. 2020; Liu et al. 2019; Kong et al. 2020). Therefore, we used the combined transcriptome and metabolome sequencing technology to analyze triploid $A$. japonicus. The different gene expression patterns of triploid and diploid A. japonicus have been identified. Some differential genes and metabolites in triploid A. japonicus that stimulate growth, metabolism, immune regulation, and protein synthesis have been found and verified, and differential genes and differential metabolites were jointly analyzed. The results in the present study will enrich the basic biological data on triploid A. japonicus, and provide resources for the future breeding of triploid $A$. japonicus.

\section{Materials and Methods}

\section{Culture and Detection of $A$. japonicus}

The triploid A. japonicus induced by hydrostatic pressure came from the same batch as the diploid control group produced at the Ministry of Agriculture and Rural Affairs' North Key Laboratory of Marine Aquaculture at Dalian Ocean University, and all were 1.5-year-old A. japonicus.
During the experiment, the breeding conditions were as follows: water temperature $14 \pm 1.5^{\circ} \mathrm{C}$, salinity $30 \pm 1$, and $\mathrm{pH}$ 7.0. During the breeding process, the water was changed every 2 days, and feeding was done once a day (feed formula: sea mud, compound feed, spirulina powder, purslane powder). We used flow cytometry (Sysmex, Japan) to determine the ploidy of A. japonicus, and the procedure was the same as Han's (Han et al. 2021).

\section{Analysis of Growth Characters}

To determine the ploidy of A. japonicus, a tiny amount of tube feet was cut and dissolved in 1.2-ml cell lysate (cystatin UV precise $\mathrm{P} / 05-5002$, Japan) in a 1.5-ml centrifuge tube. Flow cytometry was used to detect A. japonicus after the cell components had been destroyed (Han et al. 2021). Flow cytometry detected 2682 A. japonicus, including 510 triploid A. japonicus and 2172 diploid A. japonicus. The body lengths of all $A$. japonicus were then measured. To make comparisons easier, we divided the body length of $A$. japonicus into three groups $(0-6 \mathrm{~cm}, 6-12 \mathrm{~cm}$, and $12-18 \mathrm{~cm})$ and compared its proportion in each group (Fig. 1) (Table S1). We compared the aestivation times of triploid and diploid A. japonicus which is defined as the time starting with the cessation of feeding, body contraction, and inactivity and ending with relaxation, activity, and feeding (Table 1).

\section{Sample Preparation}

In 2020, three A. japonicus were dissected in each experimental group, yielding a total of 22 groups of samples, 6 of which were subjected to transcriptome sequencing (3 triploid A. japonicus, 3 diploid A. japonicus) and 16 of which were subjected to metabolomic analysis ( 8 triploid A. japonicus, 8 diploid $A$. japonicus). The body wall tissues were quickly frozen in liquid nitrogen and kept at $-80{ }^{\circ} \mathrm{C}$ in a refrigerator.

\section{RNA Extraction and Quality Control}

Total RNA was extracted from the body wall tissue of the $A$. japonicus using a Mirvana ${ }^{\mathrm{TM}}$ miRNA Isolation Kit, Ambion (USA), and the purity and concentration of total RNA were detected using the Agilent 2100 and Nanodrop (Severino et al. 2013). RNA detected with a purity between 2.0 and 2.1 was stored at $-80^{\circ} \mathrm{C}$. Gene expression was measured by the FPKM value (Li et al. 2021; Han et al. 2021).

\section{RNA-Seq and Screening for Key Differential Genes and Data Analysis}

The Cutadapt program (https://cutadapt.readthedocs.io/ en/stable/,version:cutadapt-1.9) was used to eliminate 
Fig. 1 Box diagram of body length (A) and body weight (B) of triploid and diploid Apostichopus japonicus

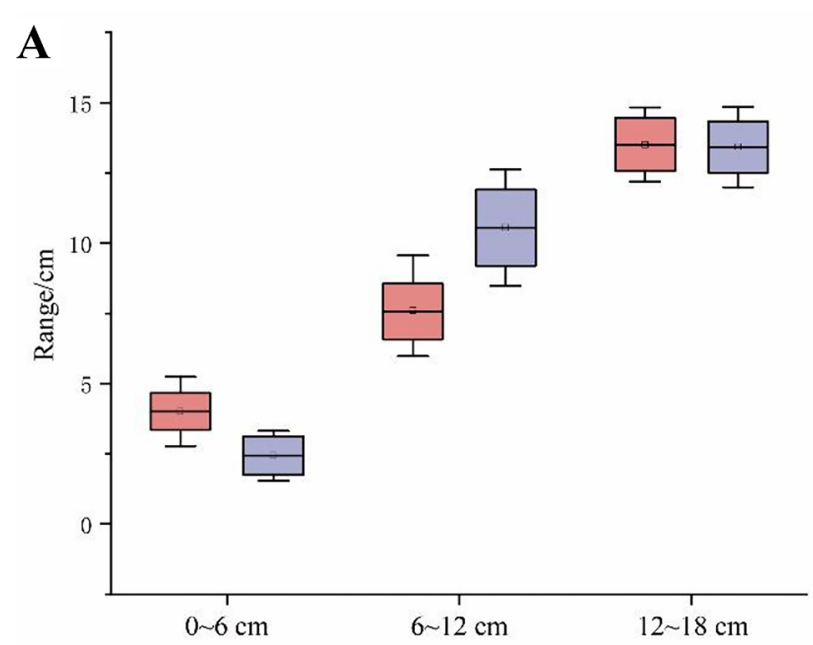

B

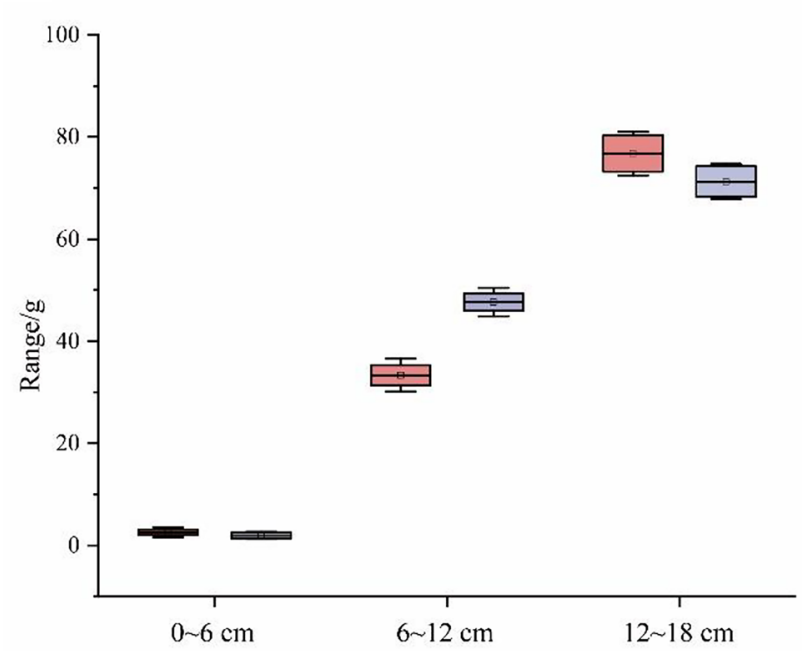

$25 \% \sim 75 \%$

I 1.5 scope within $\mathrm{IQR}$

- Median line

Mean value

- Exception value

Diploid Apostichopus japonicus

Triploid Apostichopus japonicus 
(https://bioconductor.org/packages/release/bioc/html/ edgeR.html) or DESeq2 (Pertea et al. 2015) (http://www. bioconductor.org/packages/release/bioc/html/DESeq2. html). We improved our understanding of the biological functions of the genes by using the Gene Ontology (GO) and Kyoto Encyclopedia of Genes and Genomes (KEGG) databases; enrichment analysis of GO and KEGG was performed using GSEA (gene set enrichment analysis) (https://www.omicstudio.cn/login). All RNA clean data were submitted to the Short Read Archive (SRA) Sequence Database at the National Center for Biotechnology Information (NCBI) (Accession No. PRJNA760261).

\section{qRT-PCR Validation and Analysis of DEGs}

Thirteen DEGs were validated by qRT-PCR to further validate the RNA-Seq results (Ge et al. 2020). Primer Premier 6.0 was used to create primer syntheses for the differential genes (Table 2). The $2^{-\Delta \Delta \mathrm{Ct}}$ method was used to calculate the relative expression of DEGs (Arocho et al. 2006).

\section{Analysis of the Metabolicome, Liquid Phase Analysis, and Mass Spectrometry}

For each sample, $100 \mathrm{mg}$ of A. japonicus body wall was weighed, ground with liquid nitrogen, and treated before loading the metabolome according to $\mathrm{Yu}$ et al. (2018). Before loading, all metabolic samples were kept at $-80{ }^{\circ} \mathrm{C}$. Ultra-high pressure liquid phase and a TripleTOF5600plus (SCIEX, UK) high-resolution mass spectrometer are used for analysis during liquid phase analysis and mass spectrometry. Its model and parameter settings are similar to those of Wang et al. (2019). Quality control (QC) samples were added before, during, and after the test. Prepare QC samples by combining the same number of samples to determine the instrument status and evaluate the stability of liquid chromatography-mass spectrometry (LC-MS/MS) (Li et al. 2019).

\section{Screening of Key Metabolites}

The $q$-value by BH correction was calculated using univariate analysis (Fold Change) and the $t$ test. PLS-DA VIP values were combined with multivariate statistical analysis to identify metabolites that were differentially expressed. Differential ions fulfilled the following criteria: ratio $\geq 2$ or ratio $\leq 1 / 2, q$-value $\leq 0.05$, and VIP $=1$ or higher. The KEGG enrichment pathway was used to investigate the importance of enrichment (Zheng et al. 2019).
Table 2 Primer sequences of the tested genes used in the quantitative RT-PCR analysis

\begin{tabular}{|c|c|}
\hline Gene name & Primer $\left(5^{\prime} \rightarrow 3^{\prime}\right)$ \\
\hline СYТВ & $\begin{array}{l}\text { F TGACAGGACCGCTACGAAAGAGG } \\
\text { R AAAGTTTTCTTGGGGCCGGAAGG }\end{array}$ \\
\hline CDK2 & $\begin{array}{l}\text { F CACTGCTGAAGGAGTTGGACCATG } \\
\text { R ATCGGCTGGAGACCTTGACTGG }\end{array}$ \\
\hline CDC45 & $\begin{array}{l}\text { F AACAGACGAAGATCACGCAACCTC } \\
\text { R CAAGTTCAGGAAGTGGCGGGATTC }\end{array}$ \\
\hline ORC1 & $\begin{array}{l}\text { F CAGTGACGATGAGGAGGAGGAGAG } \\
\text { R GGAGTTGCTGCTTTAGCGGAGAC }\end{array}$ \\
\hline GAMT & $\begin{array}{l}\text { F GGAAGGGGAAGACTGTAAGAGC } \\
\text { R AATAATACGCACAAGAGGCAGG }\end{array}$ \\
\hline UGT & $\begin{array}{l}\text { F GGCAGTGTTGGATCCGTTGATGG } \\
\text { R CGGTGGTGAAGTCGGCATTGG }\end{array}$ \\
\hline PGM & $\begin{array}{l}\text { F GCAGCAGCCAATCAGGTGAGG } \\
\text { R GTGAGTATAATGCCGCCGGTAGC }\end{array}$ \\
\hline MRPs & $\begin{array}{l}\text { F TCTTAGACAACGGGTGGCAAT } \\
\text { R AAGAATGTCGGGTGGTCCTG }\end{array}$ \\
\hline GP96 & $\begin{array}{l}\text { F CAAGTCGAGGAGGATGGTGAAAGC } \\
\text { R CCAGCAAAGGCAGCGGAGTC }\end{array}$ \\
\hline HSC70 & $\begin{array}{l}\text { F GCCTACCAGAGAATTGCCACATCC } \\
\text { R ACATCGGGCACTCTTTGTTCTACC }\end{array}$ \\
\hline HK & $\begin{array}{l}\text { F CGGGGAAGTAATTTCAGAGTCC } \\
\text { R ACGGCAGCGATCAATGCT }\end{array}$ \\
\hline CBRs & $\begin{array}{l}\text { F TCACAGGTTCCAACAAGGGC } \\
\text { R CTGAGTACATCTCCCCTCTGCC }\end{array}$ \\
\hline $\begin{array}{l}\text { Histidine } \\
\text { methyltransferase }\end{array}$ & $\begin{array}{l}\text { F TCAATACGAAAGCCACCAAATG } \\
\text { R CTTTACCGTCCTCTTTCGTCG }\end{array}$ \\
\hline DPD & $\begin{array}{l}\text { F TCCAGATGCCTCAAGTGCG } \\
\text { R CAATCCACTGTCCGTCATCGT }\end{array}$ \\
\hline
\end{tabular}

\section{Combined RNA-Seq and Metabolome Analysis}

The process of discovering transcriptome and metabolome associations can be broken down into three steps: (1) the KEGG metabolic pathway connects the transcriptome and metabolome, (2) data screening for statistically significant differences in data and regulatory relationships, and (3) GO and KEGG enrichment analysis.

\section{Results and Analysis}

\section{Growth Characteristics of Triploid and Diploid $A$. japonicus}

The flow cytometer results showed that the peak value of diploid A. japonicus was around 200 and that of triploid $A$. japonicus was around 300 (Fig. S6). This outcome is consistent with that of Han (Han et al. 2021). The measurement results revealed that the proportions of triploid A. japonicus in the 12-18- and 6-12-cm groups were higher than those in the diploid control group (Fig. 1 and Table S1). Aestivation 


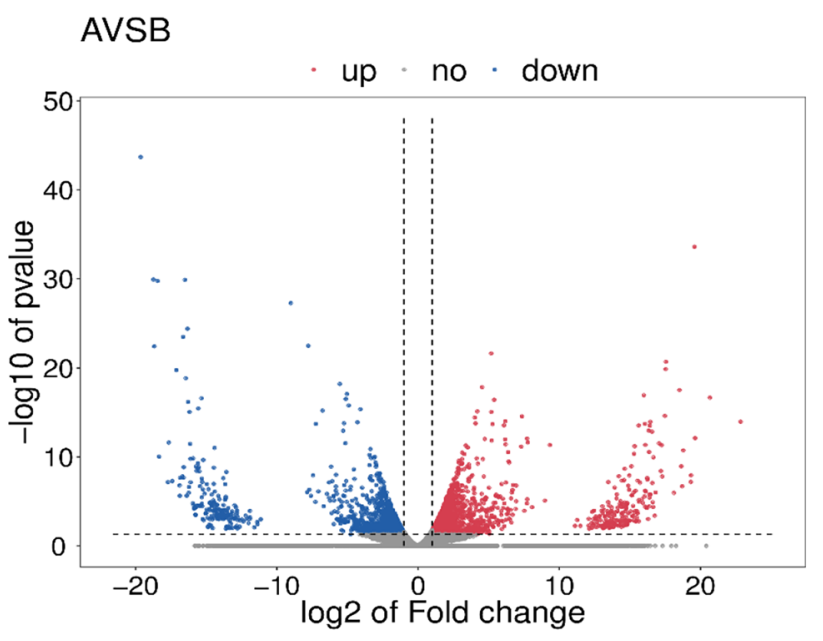

Fig. 2 Volcano plot of differentially abundant genes. Note that the red dots represent up-regulation and the blue dots represent down-regulation. A Triploid Apostichopus japonicus and B diploid Apostichopus japonicus
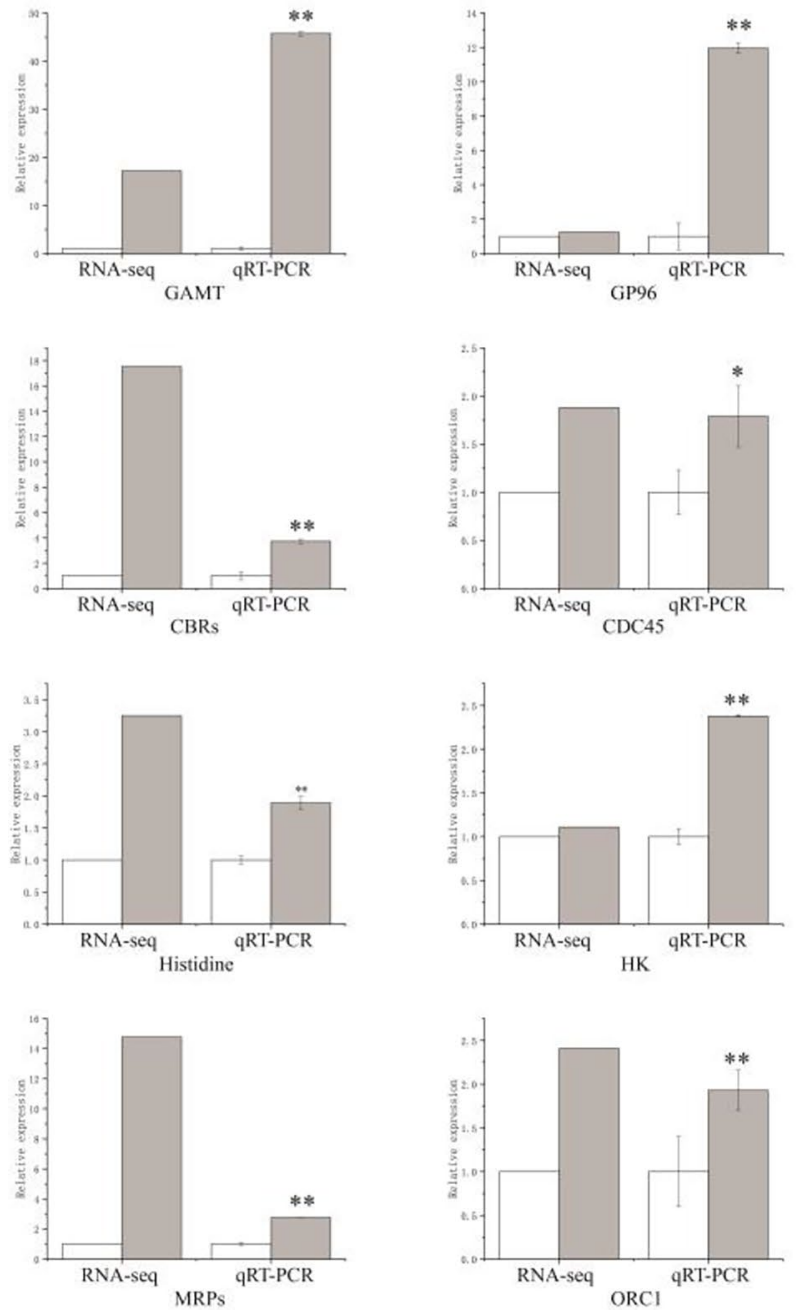

of triploid A. japonicus cultured in 2016 was observed. The results showed that aestivation duration of triploid $A$. japonicus in 2019 was 38 days, while that of diploid A. japonicus in the control group was 55 days. The triploid A. japonicus aestivation duration in 2020 was 39 days, while that of diploid A. japonicus in the control group was 63 days (Table 1). According to these data, triploid A. japonicus had a shorter aestivation time and greater growth advantage.

\section{Overview of Transcriptome Sequencing}

The transcriptome data obtained were analyzed. All samples were sequenced independently. In diploid A. japonicus, 117.5-M raw reads were obtained, while in triploid $A$. japonicus, 141.83-M raw reads were obtained. The diploid obtained 106.79-M clean reads after removing the redundant data, while the triploid obtained 125.24-M clean reads. Diploid A. japonicus transcriptome Q30 was $98.01 \%$, and
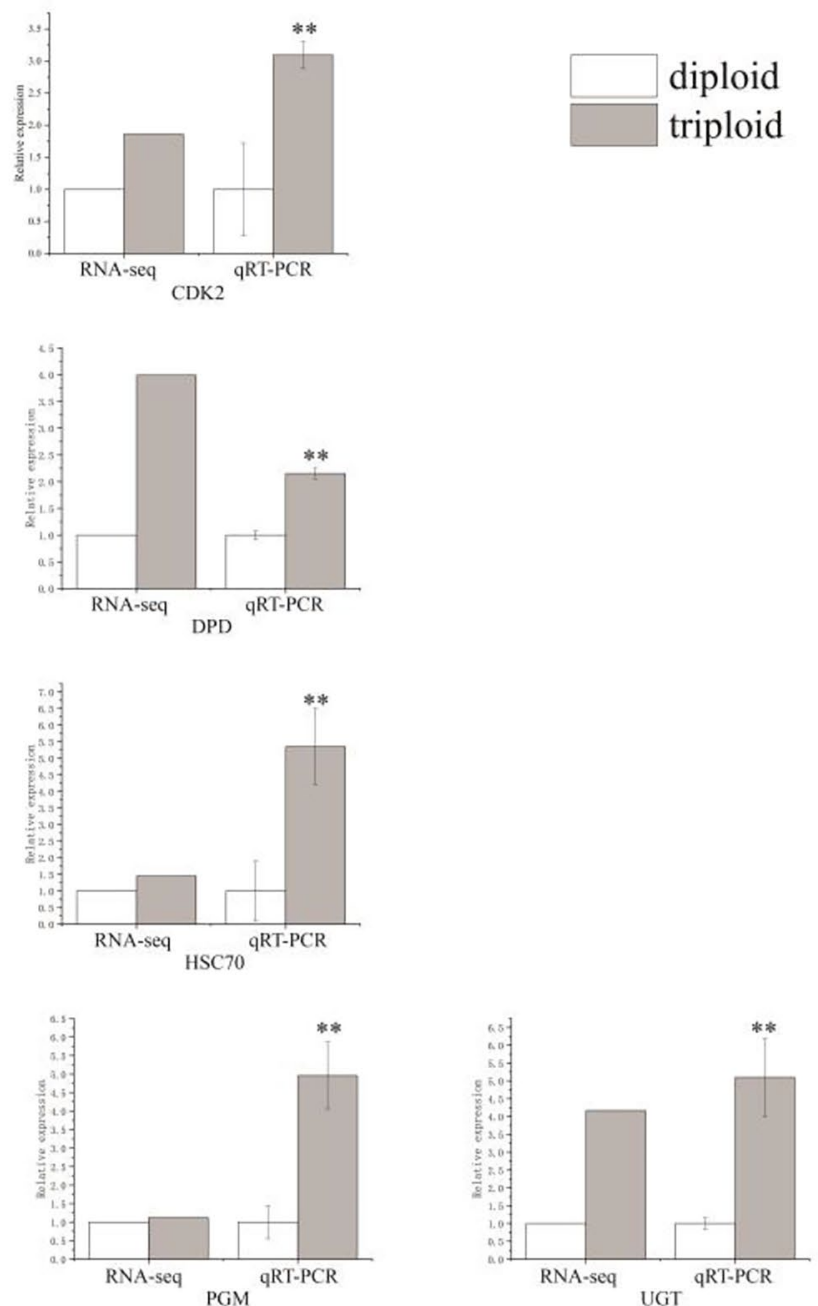

Fig. 3 Quantitative RT-PCR validation of differentially expressed genes. *Significant differences at $p<0.05$ vs. diploid (triploid). **Highly significant differences at $p<0.01$ vs. diploid (triploid). The results are expressed as mean \pm SEM and one-way ANOVA 
triploid A. japonicus transcriptome Q30 was $98.16 \%$, demonstrating the reliability of transcriptome data. When the GC content of diploid and triploid was compared, it was discovered that diploid had a GC content of $41-43 \%$ and triploid had a GC content of $42 \%$ (Table S2). A total of 3296 differential genes were identified, with 1856 being upregulated and 1440 being down-regulated (Fig. 2).

\section{DEG Enrichment Results in GO and KEGG}

The top 50 GO terms with the highest enrichment of differential genes were enriched and analyzed (Fig. S1). The results revealed that the most significantly enriched GO term in the cellular component was cytoplasmic, with 348 DEGs (up: 244, down: 104). The nucleus was the second most important location, with 314 DEGs (up: 233, down: 81). Among the molecular functions, the ATP binding process had the most differential genes (172 DEGs) (up: 126, down: 46). The second process was metal ion binding, which had 144 DEGs (up: 79, down: 65). The oxidation-reduction process was the most important item in the biological process classification, with 96 DEGs (up: 55, down: 41). Proteolysis was the second most important process, accounting for 90 DEGs in total (up: 36, down: 54).

\section{Sample class $\rightarrow$ A $\rightarrow$ B}

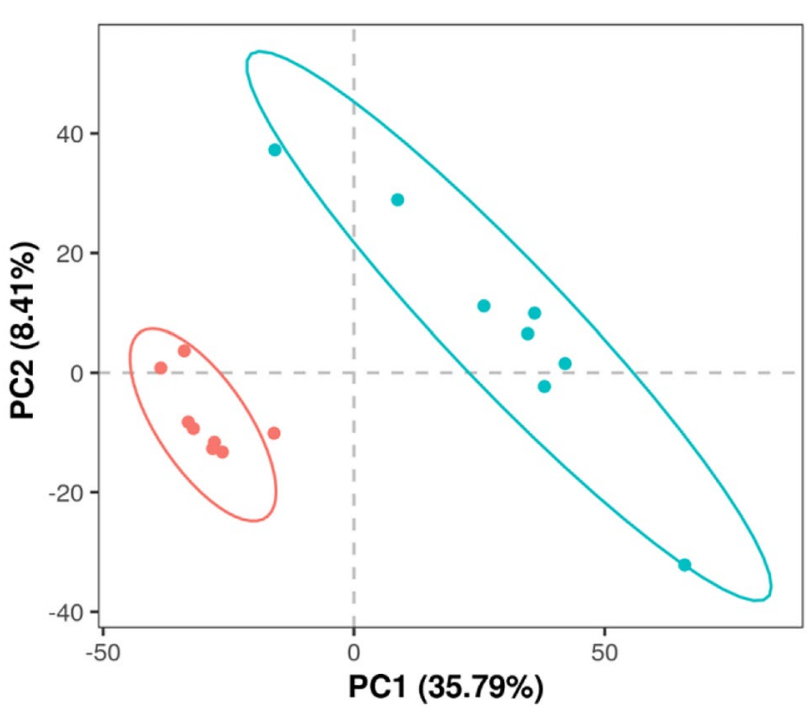

Fig. 4 Comparison group PLS-DA analysis. Note that each point in the figure represents a sample, and the similarities and differences among all samples are reflected in the tendency of separation and aggregation of samples in the figure. The aggregation of points indicates that the observed variables have a high degree of similarity, while the dispersion of points indicates that the observed variables have obvious differences. A Triploid Apostichopus japonicus and B diploid Apostichopus japonicus
Pathway items with more than 21 differential genes were screened, and the top 20 were chosen based on the $-\log 10$ $p$-value corresponding to each item in descending order. Through differential gene screening and enrichment analysis, 1114 differential genes were discovered to be enriched in 224 KEGG signaling pathways, 32 of which were significant $(p<0.05)$. DEGs were significantly enriched in pathways related to ribosome biogenesis in eukaryotes (62 DEGs; up: 57, down: 5), protein processing in the endoplasmic reticulum (52 DEGs; up: 45, down: 7), ECM-receptor interaction (50 DEGs; up: 21, down: 29), RNA transport (44 DEGs; up: 41, down: 3), and lysosomes (43 DEGs) (Fig. S2).

\section{Screening for Key Differential Genes}

According to the gene functions annotated by GO and KEGG, we discovered 13 key significantly different genes that may participate in the mechanism of growth advantage in triploid A. japonicus, and 13 genes were up-regulated, namely, cyclin-dependent kinase 2 (CDK2), cell division cycle 45 (CDC45), origin recognition complex subunit 1 (ORC1), multi-resistance associated proteins (MRPs), hexokinase (HK), UDP glucuronosyltransferase (UGT), phosphoglucomutase (PGM), carbonyl reductase (CBRs), guanidinoacetate methyltransferase (GAMT), glycoprotein 96 (GP96), heat-shock cognate protein 70 (HSC70), histidine methyltransferase, and dihydropyrimidine dehydrogenase (DPD) (Figs. 3 and 7; Table 2).

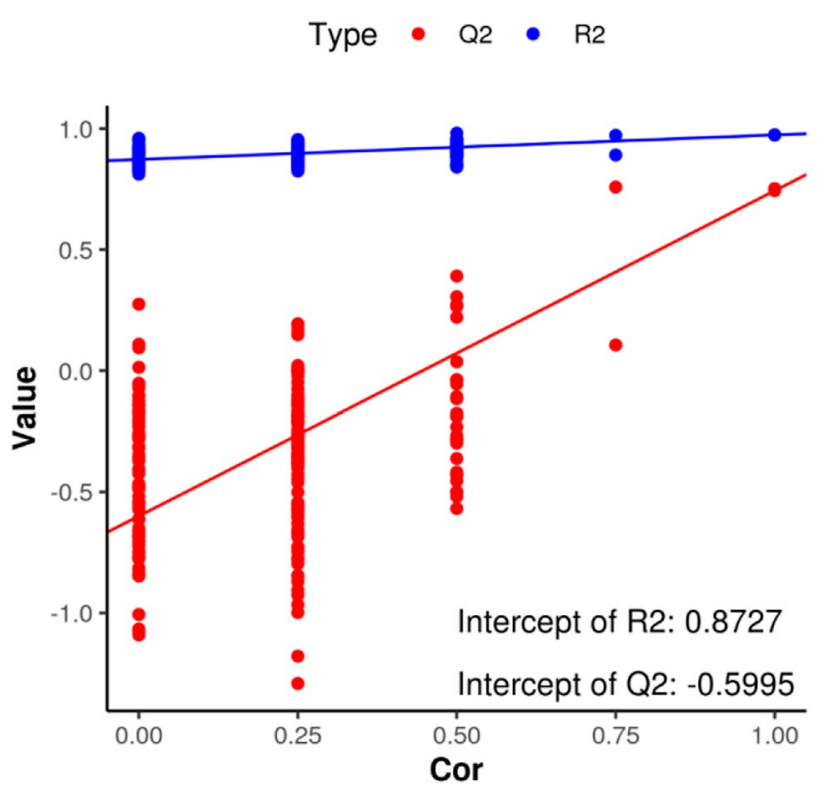

Fig. 5 Triploid and diploid Apostichopus japonicus replacement test. Note that $Q_{2}$ represents the prediction rate of the model and $R_{2}$ represents the interpretation rate of the model. $Q_{2}=-0.56510<0$, $R_{2}=0.8762$ 
Fig. 6 Volcano plot of differentially abundant metabolites. Note that the red dots represent up-regulation and the green dots represent down-regulation. A Triploid Apostichopus japonicus and $\mathbf{B}$ diploid Apostichopus japonicus

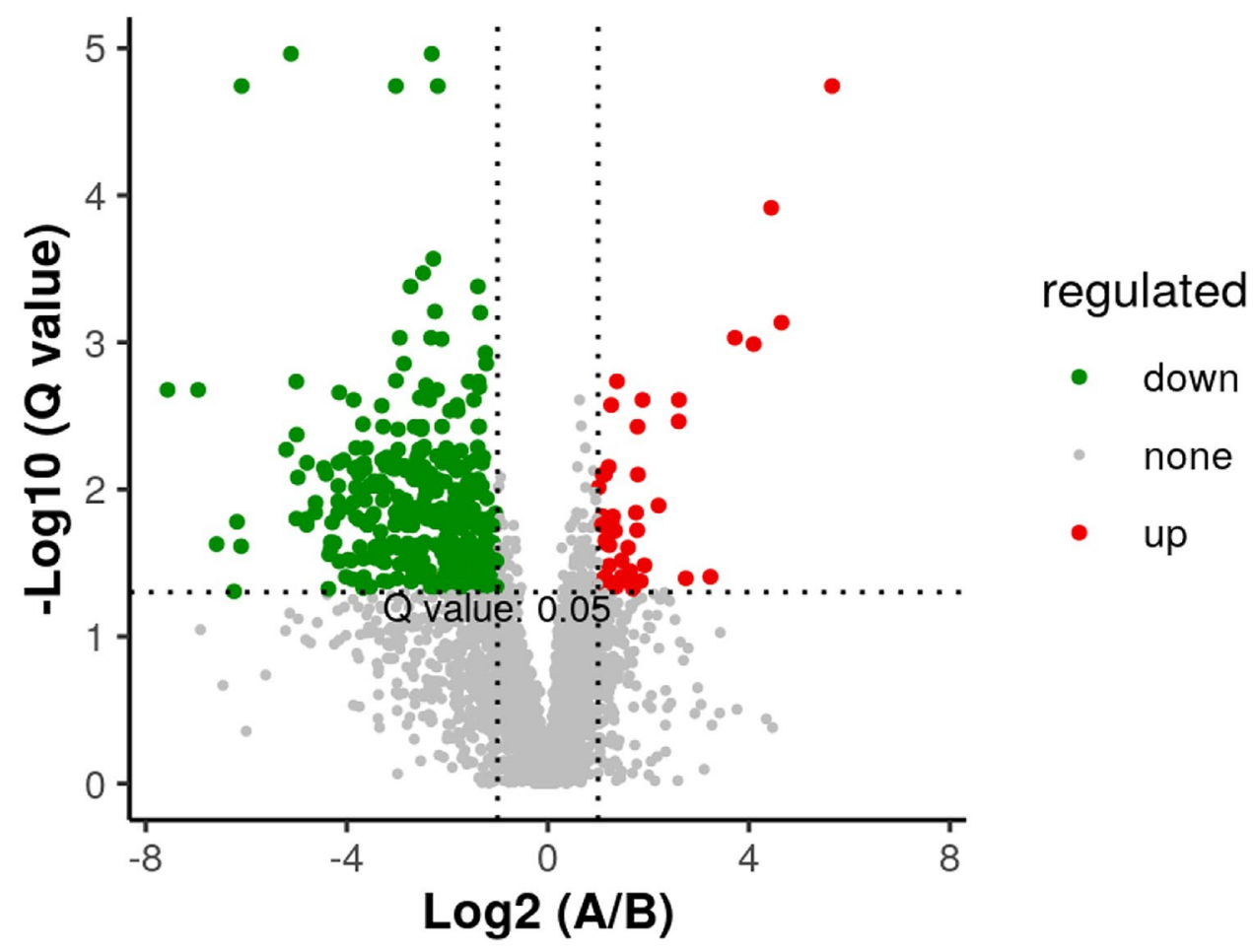

\section{qRT-PCR Validation}

The 13 key DEGs identified by transcriptome analysis were validated using qRT-PCR (Table 2). The verification results agreed with the RNA-Seq results (Fig. 3), demonstrating the reliability of the experimental results.

\section{Partial Least Squares Discriminant Analysis (PLS-DA) of Comparative Groups}

A PLS-DA model was established between each pair of groups using VIP $\geq 1.0$ as the screening condition in order to perform principal component analysis on the identified metabolic ions (Fig. 4). In this study, samples from the triploid and control groups were found to have a high degree of dispersion, with no overlap between the two groups of samples. The results were reliable, and the samples were analyzed further by metabolome.

During model validation, linear regression was performed between the original classification $Y$ matrix and the $Y$ matrix of $N$ different permutations with $R_{2} Y$ and $Q_{2} Y$, and the obtained regression line and $Y$-axis intercept values were $R_{2}$ and $Q_{2}$, respectively. They were used to determine whether the model was over-fitting. $Q_{2}=-0.56510<0, R_{2}=0.8762$. This demonstrated that the model did not involve over-fitting and that the differential metabolite analysis was sufficiently accurate (Fig. 5).

\section{Differential Metabolite Statistical Analysis and Screening}

A total of 414 metabolites were identified, including 306 positive metabolites (up: 44, down: 262) and 108 negative metabolites (up: 6, down: 102).

We discovered 11 key significantly different metabolites (SDMs). Nocodazole, lactose, lactulose, gentiobiose, hypoxanthine (HX), 2-oxoglutarate, rhododendrin, arginine, uridine, spongouridine, and aspartame were among the SDMs (Figs. 6 and 7).

\section{KEGG Pathway Enrichment Results}

According to the KEGG results, a total of 97 metabolic pathways were enriched. Significantly enriched in metabolic pathways (SDMs: 578, POS: 505, NEG: 73), porphyrin and chlorophyll metabolism (SDMs: 6, POS: 6, NEG: 0), fatty acid metabolism (SDMs: 9, POS: 8, NEG: 1), amino sugar and nucleotide sugar metabolism (SDMs: 14, POS: 14, NEG: 0), and fructose and mannose metabolism (SDMs: 6, POS: 4, NEG: 2) (Fig. S3). 


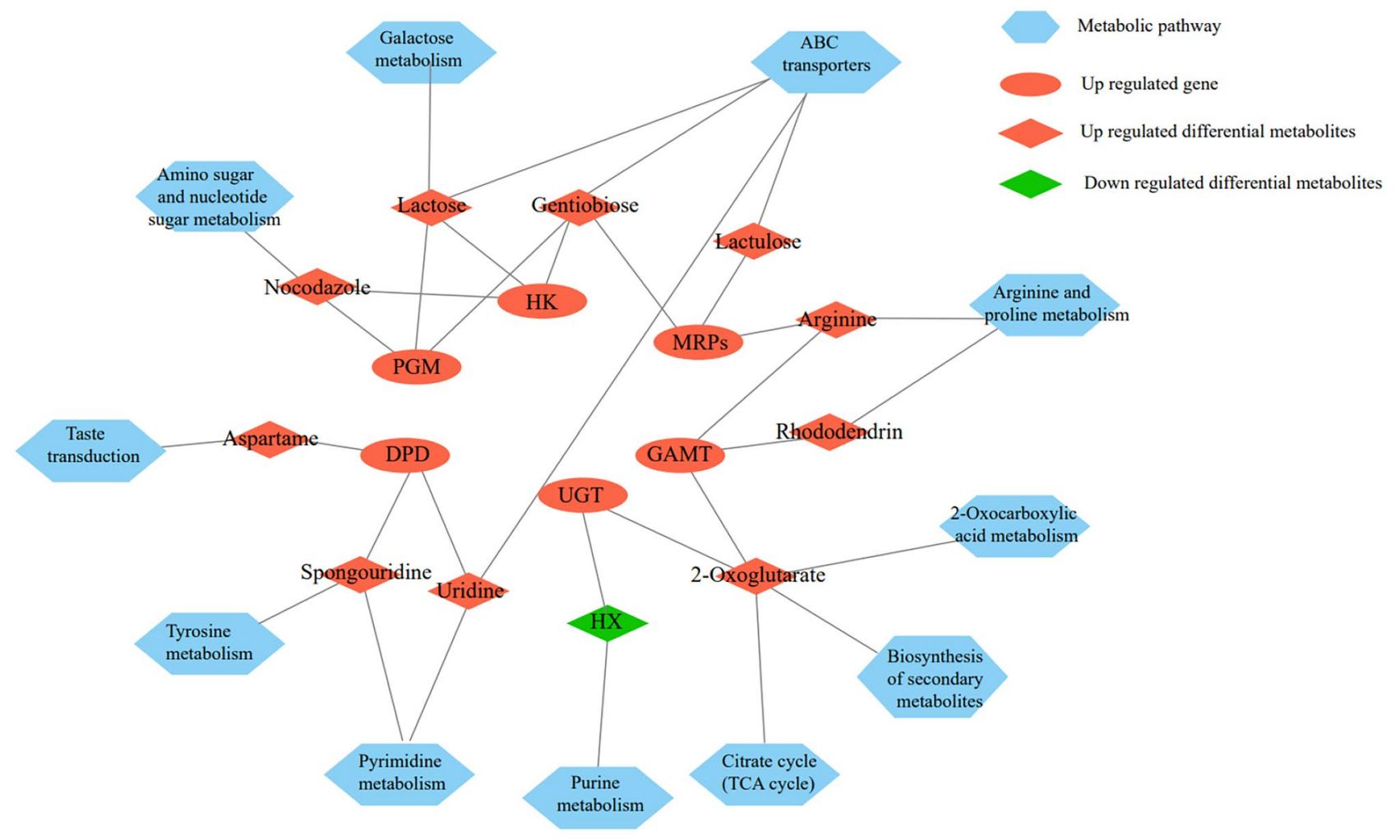

Fig. 7 Results of combined transcriptomic and metabolomic analyses

\section{Combined RNA-Seq and Metabolome Analysis Results}

We discovered that 6 DEGs may have regulatory relationships with 11 SDMs using a combined RNA-Seq and metabolome analysis, with 1 pair having negative regulation and 10 pairs having positive regulation (Fig. 7). We used GO enrichment analysis to look at the target genes of different metabolites. A total of 404 target genes (POS: 235, NEG: 165) were found to be enriched in 1151 items (POS: 666, NEG: 485). There were 572 items in terms of biological processes, mostly oxidation reduction processes (56 DEGs, POS: 28, NEG: 28) and metabolic processes (38 DEGs, POS: 20, NEG: 18), among others. There were 459 items in terms of molecular functions, mostly oxidative enzyme activity (40 DEGs, POS: 20, NEG: 20) and catalytic activity (44 DEGs, POS: 25, NEG: 19), among others. There were 120 cellular components, mostly cytosol (56 DEGs, POS: 30, NEG: 26) and cytoplasm (70 DEGs, POS: 50, NEG: 20), among others (Fig. S4); KEGG enrichment analysis of differential metabolite target genes revealed that 417 (POS: 246, NEG: 171) genes were enriched in 181 (POS: 92, NEG: 89) pathways, with purine metabolism (82 DEGs, POS: 41 , NEG: 41) and pyrimidine metabolism (58 DEGs, POS: 33, NEG: 25) being the most enriched (Fig. S5).

\section{Discussion}

The length of A. japonicus is an important economic characteristic. In this study, the length of diploid and triploid $A$. japonicus was significantly different under the same culture conditions. Among A. japonicus with a body length of 6-12 cm, the percentage of triploid A. japonicus was 4.46\% higher than that of diploid A. japonicus. Among A. japonicus with a body length of $12-18 \mathrm{~cm}$, the percentage of triploid A. japonicus was $1.37 \%$ higher than that of diploid A. japonicus. This was consistent with the triploid breeding results of other aquatic species (Peruzzi et al. 2018; Liu et al. 2018; Garnier-Géré et al. 2002). Following a comparative analysis, the aestivation time of triploid A. japonicus was shorter. This study proved that triploid A. japonicus had a better growth advantage.

Following sequencing analysis, we found that 6 DEGs had regulatory relationships with 11 SDMs, which jointly acted on 11 metabolic pathways (Fig. 7). The observed potential candidate genes mainly involved functions such as promoting growth and development, immune regulation, accumulation of carbohydrate, energy storage, and synthesis of beneficial metabolites; the differential metabolites were mainly concentrated in functions such as growth, immunity, carbohydrate synthesis, and taste improvement. 
The results showed that triploid A. japonicus had certain growth advantages. We investigated whether the significant growth advantage of triploid A. japonicus was related to the enhancement of cell division, as the expressions of CDK2, CDC45, ORC1, and histidine methyltransferase were significantly higher in triploids. $C D K 2$ plays a key role in cell cycle regulation and participates in a series of biological processes. Existing research shows that $C D K 2$ may be involved in DNA damage and phosphorylation of protein interactions and antitumor activity (Tadesse et al. 2020; Spencer et al. 2013). CDC45 can participate in the formation of the eukaryotic replication helicase and play an important role in the initial stage of DNA replication; thus, it can promote the cell cycle (Rios-Morales et al. 2019). Research has shown that DNAJAl after being activated by stable CDC45 promotes the cell cycle (Yang et al. 2020). $O R C l$ can participate in eukaryotic replication and maintain genome stability (De et al. 2019). The studies by Okano have proved that $\mathrm{ORCl}$ is essential for cell mitosis (OkanoUchida et al. 2018). Histidine methyltransferase can regulate the differentiation of muscle cells and promote the growth and development of A. japonicus (Shu and Du 2021). The participation of genes in immune regulation contributes to the growth of triploid A. japonicus; the expressions of $U G T$ , GP96, and HSC70 are much higher in triploids. UGT is a glycoprotein attached to the endoplasmic reticulum cavity of microsomes. Bigo et al. (2013) indicated that UGT acted as a key metabolic protein in organisms to hinder the accumulation of toxic hydrophilic compounds. Primarily, it catalyzes the combination of endogenous or exogenous compounds with the cofactor uridine diphosphate glucuronic acid, thereby enhancing the polarity of lipophilic substrates and facilitating their excretion via urine or bile. For this reason, it has been proposed that triploid A. japonicus has a higher immunity and stronger stress resistance. The two heat shock proteins, GP96 and $H S C 70$, are generated at abnormal temperatures in an environment with extremely low oxygen content and oxidized free radicals during the bacterial infection process. They are able to repair denatured proteins, accelerate the recovery of normal proteins, and strongly protect cells when exposed to stress (Zininga et al. 2018). High temperature can induce the high expression of $\mathrm{HSC} 70$. The up-regulated expression of $H S C 70$ gene can protect against injury due to high temperature (Sun et al. 2016). GP96 is highly expressed following increased temperature; therefore, it can resist high temperature (Tang et al. 2009). This may be the reason why the aestivation time of triploid A. japonicus is shorter. Also, they are important players in the processing of proteins in the endoplasmic reticulum (Cosin-Roger et al. 2018; Stricher et al. 2013). HSC70 promote the processing and synthesis of proteins and benefits the growth of organisms (Liu et al. 2012); thus, it could be speculated that triploid A. japonicus has potential immune regulation ability than diploid A. japonicus. DPD is an enzyme mainly involved in the metabolism of pyrimidines in organisms. It contains the ability to metabolize the toxic pyrimidine analogue fluorouracil (5-FU). DPD is the rate limiting enzyme of fluoropyrimidine metabolism and can convert 5-FU and its metabolites into non-cytotoxic products and excrete them out of the body (Sharma et al. 2019). DPD expression was increased in triploid A. japonicus. Therefore, we speculate that triploid A. japonicus has a better ability to excrete toxins.

Carbohydrate accumulation can provide energy for triploid A. japonicus, and its related genes are $H K$ and $P G M$, which are significantly up-regulated. $H K$ is a pivotal rate-limiting enzyme in glycolysis; it is able to stimulate the utilization of glucose by cells and performs an extremely important function in energy metabolism (Patra et al. 2013). The energy provided by $H K$ up-regulation may be used to resist the adverse effects at high temperature (Scaraffia and Gerez 2000). Variations in $H K$ activity will give rise to changes in glycolysis and the formation of pentose phosphates, nicotinamide adenine dinucleotide phosphate hydrogen (NADPH), and glycogens (Anderson et al. 1971). $H K$ with high activity is therefore beneficial to allow it to tackle hypoxia and high temperature stress. $P G M$ participates in glycolysis and gluconeogenesis in vivo. Fragmentary or missing $P G M$ will affect the synthesis of some elements of the cell wall (Morava 2014), and PGM deficiency greatly influences the glycosylation of proteins (Beamer 2015). Hence, high expression levels of related genes are speculated to be a vital influencing factor on the rapid growth and development of triploid A. japonicus. These results showed that the genes related to energy metabolism were up-regulated. MRPs and GAMT were significantly up-regulated in triploid A. japonicus. MRPs and GAMT are all related to the ATP energy supply. MRPs are members of the subfamily $\mathrm{C}$ of the $\mathrm{ABC}$ transporter superfamily and serve as efflux pumps for ATP (Zhang et al. 2015). They can pump antitumor drugs conjugated to glutathione, glucuronate, or sulfate out of cells. Arginine can generate creatine and glycocyamine under the action of GAMT. Studies have shown that creatine plays an important role in the conversion of ATP and participates in stabilizing a form of ATP (Joncquel-Chevalier et al. 2015). It is speculated that the up-regulation of MRPS and GAMT promotes the energy supply of triploid A. japonicus. Genes related to the synthesis of beneficial metabolites were up-regulated. $C B R s$ is a type of oxidoreductase protein, which is widely found in bacteria, fungi, yeasts, animals, and plants. With coenzyme $\mathrm{NAD}(\mathrm{P})+$ and $\mathrm{NAD}(\mathrm{P}) \mathrm{H}$ as the electron acceptor and donor, respectively, $C B R s$ can specifically catalyze the interconversion between ketone (aldehyde) and alcohol, and contribute to the synthesis of valuable hydroxy compounds and metabolites (Forrest and Gonzalez 2000). 
According to the combined analysis of transcriptome and metabolome, we screened 11 key SDMs which are related to growth and development (SDMs: nocodazole, rhododendrin, 2-oxoglutarate, arginine, lactulose, and HX), immunity (SDMs: uridine and spongouridine), and taste (SDMs: lactose, gentiobiose, and aspartame), respectively. Nocodazole, rhododendrin, 2-oxoglutarate (AKG), arginine and lactulose are positively regulated by the DEGs $H K$ (SDM: nocodazole), PGM (SDM: nocodazole), GAMT (SDMs: rhododendrin and arginine), and MRPs (SDMs: arginine and lactulose). $\mathrm{HX}$ is negatively regulated by $U G T$ . They are involved in the metabolic pathways of amino sugar and nucleotide sugar metabolism, arginine and proline metabolism, TCA cycle, 2-oxocarboxylic acid metabolism, biosynthesis of secondary metabolites, $\mathrm{ABC}$ transporters, and purine metabolism. Nocodazole is a mitotic blocker in the same class as colchicine, and it can induce the arrest of cell division in the $M$ phase and lead to cell synchronization (Cooper et al. 2006). Attia found that nocodazole was a germ cell mutagen, which induced mutation lethality in male germ cells (Attia et al. 2015). Based on its ability to kill germ cells, it was inferred that the up-regulated expression of such metabolites is related to the sterility of triploid A. japonicus. According to research carried out by Kim (Kim et al. 2019), rhododendrin significantly enhanced the activity of dopaminergic neurons. The metabolite AKG can promote growth. Studies have shown that AKG can promote the synthesis of muscle protein (Pierzynowski and Sjodin 1998). In piglets with slow perinatal growth, AKG as an energy donor can promote bone metabolism (Tomaszewska et al. 2021). Arginine is a basic component of various proteins, and its precursor can regulate cell proliferation, differentiation, and homeostasis (Bulau et al. 2006). Research carried out by Deng indicated that lactulose can promote enterocinesia (Deng et al. 2021). It may accelerate the intestinal absorption of nutrients and provide more energy for the body. HX is an important purine alkaloid that degrades fats. The down-regulation of HX benefits the development of triploid A. japonicus. Therefore, these metabolites may lead to the rapid growth and development of triploid A. japonicus. Uridine and spongouridine are positively regulated by $D P D$, and they are jointly involved in the metabolic pathways of $\mathrm{ABC}$ transporters, pyrimidine metabolism, and tyrosine metabolism. Uridine is a ribose extracted from nucleic acids. Walker revealed that uridine reduced cell apoptosis, and prevented mitochondrial DNA deletion and mitochondrial depolarization (Walker and Venhoff 2005). It has been found that spongouridine, a metabolite with antibacterial ability, inhibited bacteria and fungi to some extent (Hamoda et al. 2021). It is speculated that the up-regulation of these metabolites can enhance the antibacterial immunity of triploid A. japonicus. Lactose, gentiobiose, and aspartame are positively regulated by the
DEGs $H K$ (SDMs: lactose and gentiobiose), PGM (SDMs: lactose and gentiobiose), and $D P D$ (SDM: aspartame). They are involved in the metabolic pathways of galactose metabolism, ABC transporters, and taste transduction. Lactose, one of the sources of dietary energy, can effectively improve the quality of products, maintain the color of products, and increase the overall sugar content without making the products too sweet and greasy. Luo and Jiang (2006) proved that lactose improved the flavor of shredded squid and reduced water activity. As a type of functional oligosaccharide, gentiobiose can effectively improve food flavors, and function as an immune regulator in vitro (İspirli et al. 2019). Aspartame is an artificial dipeptide sweetener that is able to affect a variety of cells and tissues (Choudhary and Pretorius 2017). It is presumed that the up-regulated expression of these metabolites can enhance the nutritional value of triploid A. japonicus, and optimize flavor and taste.

Compared with diploid A. japonicus, triploid A. japonicus had more advantages in terms of gene regulation ability, error-tolerant rate of gene expression, immunity, growth and development, stress resistance, energy conversion rate, adaptability to a harsh living environment, edible value, and taste. Our results further enrich the biological data of triploid A. japonicus and provide useful resources for genetic improvement of this species.

Supplementary Information The online version contains supplementary material available at https://doi.org/10.1007/s10126-022-10093-4.

Author Contribution YQC and JD designed and conceived the whole experiments. JHX and YS collected important background information. JHX, YS, YC, LSH, BCD, YXL, CG, PFH, XJ, JS, and DHY performed the experiments. JHX and YS carried out literature retrieval, data collection, and data analysis. YC and LSH provided help for data analysis. JHX, YS, and JD wrote the paper. YQC ang JD checked the manuscript. All authors read and approved the final manuscript.

Funding This work was funded by the National Natural Science Foundation of China (No. 31772849), Liaoning Provincial Department of Education in 2019 (DL201901), Program for Liaoning Innovative Research Team in University (LT2019003), and High-level talent support grant for innovation in Dalian (2020RD03).

\section{Declarations}

Conflict of Interest The authors declare no competing interests.

Open Access This article is licensed under a Creative Commons Attribution 4.0 International License, which permits use, sharing, adaptation, distribution and reproduction in any medium or format, as long as you give appropriate credit to the original author(s) and the source, provide a link to the Creative Commons licence, and indicate if changes were made. The images or other third party material in this article are included in the article's Creative Commons licence, unless indicated otherwise in a credit line to the material. If material is not included in the article's Creative Commons licence and your intended use is not permitted by statutory regulation or exceeds the permitted use, you will 
need to obtain permission directly from the copyright holder. To view a copy of this licence, visit http://creativecommons.org/licenses/by/4.0/.

\section{References}

Anderson JW, Herman RH, Tyrrell JB, Cohn RM (1971) Hexokinase: a compartmented enzyme. Am J Clin Nutr 24:642-650

Arocho A, Chen B, Ladanyi M, Pan Q (2006) Validation of the 2-DeltaDeltaCt calculation as an alternate method of data analysis for quantitative PCR of BCR-ABL P210 transcripts. Diagnos Mole Pathol: Am J Surg Pathol Part b 15:56-61

Attia SM, Ahmad SF, Okash RM, Bakheet SA (2015) Dominant lethal effects of nocodazole in germ cells of male mice. Food Chem Toxicol: an Int J Pub for the British Industr Biolog Res Associat 77:101-104

Beamer LJ (2015) Mutations in hereditary phosphoglucomutase 1 deficiency map to key regions of enzyme structure and function. J Inherit Metab Dis 38:243-256

Bigo C, Caron S, Dallaire-Théroux A, Barbier O (2013) Nuclear receptors and endobiotics glucuronidation: the good, the bad, and the UGT. Drug Metab Rev 45:34-47

Bulau P, Zakrzewicz D, Kitowska K, Wardega B, Kreuder J, Eickelberg O (2006) Quantitative assessment of arginine methylation in free versus protein-incorporated amino acids in vitro and in vivo using protein hydrolysis and high-performance liquid chromatography. Biotechniques 40:305-310

Carmona SJ, Teichmann SA, Ferreira L, Macaulay IC, Stubbington MJ, Cvejic A, Gfeller D (2017) Single-cell transcriptome analysis of fish immune cells provides insight into the evolution of vertebrate immune cell types. Genome Res 27:451-461

Chang Y, Xiang J (2002) Inducement of polyploidy sea cucumber (Apostichopus japonicus Liao). J Dalian Ocean Univ 17:1-7

Chen SA, Hou J, Yao N, Xie C, Li D (2020) Comparative transcriptome analysis of Triplophysa yarkandensis in response to salinity and alkalinity stress. Comparative biochemistry and physiology. Part D Genom Proteom 33:100629

Choudhary AK, Pretorius E (2017) Revisiting the safety of aspartame. Nutr Rev 75:718-730

Cooper S, Iyer G, Tarquini M, Bissett P (2006) Nocodazole does not synchronize cells: implications for cell-cycle control and wholeculture synchronization. Cell Tissue Res 324:237-242

Cosin-Roger J, Spalinger MR, Ruiz PA, Stanzel C, Terhalle A, Wolfram L, Melhem H, Atrott K, Lang S, Frey-Wagner I, Fried M, Scharl M, Hausmann M, Rogler G (2018) Gp96 deficiency affects TLR4 functionality and impairs ERK and p38 phosphorylation. PLoS One 13:e0193003

De IP, Leon VA, Kuang Z, Wang M, Boeke JD, Hochwagen A, Armache $\mathrm{KJ}$ (2019) Structure and function of the Orc1 BAH-nucleosome complex. Nat Commun 10:2894

Deng Z, Fu Z, Yan W, Nie K, Ding L, Ma D, Huang H, Li T, Xie J, Fu L (2021) The different effects of Chinese herb solid drink and lactulose on gut microbiota in rats with slow transit constipation induced by compound diphenoxylate. Food Res Int 143:110273

Ding J, Chang Y, Wang Z, Song J (2007) Polyploidy induction by hydrostatic pressure shock and embryo development of sea cucumber Apostichopus japonicus. Chin J Oceanol Limnol 25:184-190

Forrest GL, Gonzalez B (2000) Carbonyl reductase. Chem Biol Interact 129:21-40

Garnier-Géré PH, Naciri-Graven Y, Bougrier S, Magoulas A, Héral M, Kotoulas G, Hawkins A, Gérard A (2002) Influences of triploidy, parentage and genetic diversity on growth of the Pacific oyster Crassostrea gigas reared in contrasting natural environments. Mol Ecol 11:1499-1514
Ge Q, Feng F, Liu L, Chen L, Lv P, Ma S, Chen K, Yao Q (2020) RNASeq analysis of the pathogenesis of STZ-induced male diabetic mouse liver. J Diabetes Complications 34:107444

Guo X (2021) Genetics in shellfish culture. In: Molluscan Shellfish Aquaculture: A Practical Guide, S. Shumway (ed.), $5 \mathrm{~m}$ Books Ltd Essex UK 552p

Guo X, Allen SK Jr (1994) Sex determination and polyploid gigantism in the dwarf surfclam (Mulinia lateralis Say). Genetics 138:1199-1206

Guo X, DeBrosse G, Allen SK Jr (1996) All-triploid Pacific oysters (Crassostrea gigas Thunberg) produced by mating tetraploids and diploids. Aquaculture 142:61

Guo X, Wang Y, Xu Z, Yang H (2009) Chromosome set manipulation in shellfish. New Technologies in Aquaculture 52:165-194

Hamoda AM, Fayed B, Ashmawy NS, El-Shorbagi AA, Hamdy R, Soliman SSM (2021) Marine sponge is a promising natural source of anti-SARS-CoV-2 scaffold. Front Pharmacol 13:666664

Han L, Sun Y, Cao Y, Gao P, Quan Z, Chang Y, Ding J (2021) Analysis of the gene transcription patterns and DNA methylation characteristics of triploid sea cucumbers (Apostichopus japonicus). Sci Rep 11:7564-7564

İspirli H, Colquhoun IJ, Şahin E, Sagdic O, Dertli E (2019) Preparation of gentiobiose-derived oligosaccharides by glucansucrase E81 and determination of prebiotic and immune-modulatory functions. Carbohydr Res 486:107837

Joana TDS, Allen JSK, Wolfe BM, Small JM (2018) Mitotic instability in triploid and tetraploid one-year-old eastern oyster, Crassostrea virginica, assessed by cytogenetic and flow cytometry techniques. Genome 61:79-89

Joncquel-Chevalier CM, Voicu PM, Fontaine M, Dessein AF, Porchet N, Mention-Mulliez K, Dobbelaere D, Soto-Ares G, Cheillan D, Vamecq J (2015) Creatine biosynthesis and transport in health and disease. Biochimie 119:146-165

Kim H, Park J, Leem H, Cho M, Yoon JH, Maeng HJ, Lee Y (2019) Rhododendrin-induced RNF146 expression via estrogen receptor $\beta$ activation is cytoprotective against 6-OHDA-induced oxidative stress. Int J Mol Sci 20:1772

Kong T, Lin S, Ren X, Li S, Gong Y (2020) Transcriptome and metabolome integration analysis of mud crab Scylla paramamosain challenged to Vibrio parahaemolyticus infection. Fish Shellfish Immunol 103:430-437

Li L, Chen M, Storey KB (2019) Metabolic response of longitudinal muscles to acute hypoxia in sea cucumber Apostichopus japonicus (Selenka): a metabolome integrated analysis. Comp Biochem Physiol Part D Genom Proteom 29:235-244

Li Y, Fang J, Qi X, Lin M, Zhong Y, Sun L, Cui W (2018) Combined analysis of the fruit metabolome and transcriptome reveals candidate genes involved in flavonoid biosynthesis in Actinidia arguta. Int J Mol Sci 19:1471

Li B, Zhang P, Wang F, Li R, Liu J, Wang Q, Liu W, Wang B, Hu G (2021) Integrated analysis of the transcriptome and metabolome revealed candidate genes involved in GA3-induced dormancy release in Leymus chinensis seeds. Int J Mol Sci 22:4161

Liu T, Daniels CK, Cao S (2012) Comprehensive review on the HSC70 functions, interactions with related molecules and involvement in clinical diseases and therapeutic potential. Pharmacol Ther 136:354-374

Liu H, Wu Z, Zhu X, Song Z, Hu J, Wang L, Li J, You F (2018) Comparative performance of growth, vertebral structure and muscle composition in diploid and triploid Paralichthys olivaceus. J Fish Dis 41:1495-1504

Liu K, Yin D, Shu Y, Dai P, Yang Y, Wu H (2019) Transcriptome and metabolome analyses of Coilia nasus in response to Anisakidae parasite infection. Fish Shellfish Immunol 87:235-242 
Luo Y, Jiang Y (2006) Application of lactose in the sea foods processing. Food Additi China 5:135-137

Morava E (2014) Galactose supplementation in phosphoglucomutase-1 deficiency; review and outlook for a novel treatable CDG. Mol Genet Metab 112:275-279

Okano-Uchida T, Kent LN, Ouseph MM, McCarty B, Frank JJ, Kladney R, Cuitino MC, Thompson JC, Coppola V, Asano M, Leone G (2018) Endoreduplication of the mouse genome in the absence of ORC1. Genes Dev 32:978-990

Patra KC, Wang Q, Bhaskar PT, Miller L, Wang Z, Wheaton W, Chandel N, Laakso M, Muller WJ, Allen EL, Jha AK, Smolen GA, Clasquin MF, Robey B, Hay N (2013) Hexokinase 2 is required for tumor initiation and maintenance and its systemic deletion is therapeutic in mouse models of cancer. Cancer Cell 24:213-228

Pertea M, Pertea GM, Antonescu CM, Chang TC, Mendell JT, Salzberg SL (2015) StringTie enables improved reconstruction of a transcriptome from RNA-seq reads. Nat Biotechnol 33:290-295

Peruzzi S, Puvanendran V, Riesen G, Seim RR, Hagen $\emptyset$, MartínezLlorens S, Falk-Petersen IB, Fernandes JMO, Jobling M (2018) Growth and development of skeletal anomalies in diploid and triploid Atlantic salmon (Salmo salar) fed phosphorus-rich diets with fish meal and hydrolyzed fish protein. PLoS One 13:e0194340

Pierzynowski SG, Sjodin A (1998) Perspectives of glutamine and its derivatives as feed additives for farm animals. J Animal Feed Sci 7:79-91

Qin Y, Zhang Y, Ma H, Wu X, Xiao S, Li J, Mo R, Yu Z (2018) Comparison of the biochemical composition and nutritional quality between diploid and triploid Hong Kong oysters. Crassostrea Hongkongensis Front Physiol 9:1674

Ren L, Gao X, Yang C, Tan H, Cui J, Wang S, Li W, Zhang C, Tao M, Qin Q, Liu S (2018) Comparison of diploid and triploid Carassius auratus provides insights into adaptation to environmental change. Sci China Life Sci 61:1407-1419

Rios-Morales RY, Chan SH, Bell SP (2019) Initiation-specific alleles of the $\mathrm{Cdc} 45$ helicase-activating protein. PLoS One 14:e0214426

Robinson MD, McCarthy DJ, Smyth GK (2010) edgeR: a Bioconductor package for differential expression analysis of digital gene expression data. Bioinformatics (oxford, England) 26:139-140

Scaraffia PY, Gerez BNM (2000) Effects of temperature and pH on hexokinase from the flight muscles of Dipetalogaster maximus (Hemiptera: Reduviidae). J Med Entomol 37:689-694

Severino P, Brüggemann H, Andreghetto FM, Camps C, Klingbeil Mde F, de Pereira WO, Soares RM, Moyses R, Wünsch-Filho V, Mathor MB, Nunes FD, Ragoussis J, Tajara EH (2013) MicroRNA expression profile in head and neck cancer: HOX-cluster embedded microRNA-196a and microRNA-10b dysregulation implicated in cell proliferation. BMC Cancer 13:533

Sharma V, Gupta SK, Verma M (2019) Dihydropyrimidine dehydrogenase in the metabolism of the anticancer drugs. Cancer Chemother Pharmacol 84:1157-1166

Shi W, Zhang J, Wang Y, Ji J, Guo L, Ren Y, Qiao G, Wang Q, Li Q (2020) Transcriptome analysis of sea cucumber (Apostichopus japonicus) polian vesicles in response to evisceration. Fish Shellfish Immunol 97:108-113

Shu WJ, Du HN (1875) (2021) The methyltransferase SETD3-mediated histidine methylation: biological functions and potential implications in cancers. Biochim Biophys Acta Rev Cancer 1875:188465

Song B, Wang H, Ye F (2004) Study on polyploid breeding technology of aquatic animals. Beij Fisher 6:38-41. CNKI:SUN:BJSI.0.2004-06-020. (InChinese)

Spencer SL, Cappell SD, Tsai FC, Overton KW, Wang CL, Meyer T (2013) The proliferation-quiescence decision is controlled by a bifurcation in CDK2 activity at mitotic exit. Cell 155:369-383
Stricher F, Macri C, Ruff M, Muller S (2013) HSPA8/HSC70 chaperone protein: structure, function, and chemical targeting. Autophagy 9:1937-1954

Sun L, Sun J, Xu Q, Li X, Zhang L, Yang H (2017) Metabolic responses to intestine regeneration in sea cucumbers Apostichopus japonicus. Comp Biochem Physiol Part D Genomics Proteomics 22:32-38

Sun Y, Zhao J, Sheng Y, Xiao Y, Zhang Y, Bai L, Tan Y, Xiao L, Xu $G$ (2016) Identification of heat shock cognate protein 70 gene (Alhsc70) of Apolygus lucorum and its expression in response to different temperature and pesticide stresses. Insect Sci 23:37-49

Tadesse S, Anshabo AT, Portman N, Lim E, Tilley W, Caldon CE, Wang S (2020) Targeting CDK2 in cancer: challenges and opportunities for therapy. Drug Discov Today 25:406-413

Tang Y, Li H, Hamel JF (2009) Significances of pH and temperature on the production of heat-shock protein glycoprotein 96 by MethA tumor cell suspension culture in stirred-tank bioreactors. Bioprocess Biosyst Eng 32:267-276

Tomaszewska E, Burmańczuk N, Dobrowolski P, Świątkiewicz M, Donaldson J, Burmańczuk A, Mielnik-Błaszczak M, Kuc D, Milewski S, Muszyński S (2021) The protective role of alphaketoglutaric acid on the growth and bone development of experimentally induced perinatal growth-retarded piglets. Animals: An Open Acc J MDPI 11:137

Wang H, Ding J, Ding S, Chang Y (2019) Metabolomic changes and polyunsaturated fatty acid biosynthesis during gonadal growth and development in the sea urchin Strongylocentrotus intermedius. Comp Biochem Physiol Part D Genomics Proteomics 32:100611

Wang H, Ding J, Ding S, Chang Y (2020) Integrated metabolomic and transcriptomic analyses identify critical genes in eicosapentaenoic acid biosynthesis and metabolism in the sea urchin Strongylocentrotus intermedius. Sci Rep 10:1697

Walker UA, Venhoff N (2005) Uridine in the prevention and treatment of NRTI-related mitochondrial toxicity. Antivir Ther 10:M117M123 (PMID: 16152713)

Winkler H (1916) Uber die experimentelle Erzeugung von Pflanzen mit abweichenden Chromosomenzahlen. Zeitschrift Für Induktive Abstammungs Und Vererbungslehre 17:270-272

Xing L, Sun L, Liu S, Zhang L, Sun J, Yang H (2021) Metabolomic analysis of white, green and purple morphs of sea cucumber Apostichopus japonicus during body color pigmentation process. Comp Biochem Physiol Part D Genomics Proteomics 39:100827

Yang S, Ren X, Liang Y, Yan Y, Zhou Y, Hu J, Wang Z, Song F, Wang F, Liao W, Liao W, Ding Y, Liang L (2020) KNK437 restricts the growth and metastasis of colorectal cancer via targeting DNAJA1/ CDC45 axis. Oncogene 39:249-261

Yu C, Luo X, Zhan X, Hao J, Zhang L, Song YB, Shen C, Dong M (2018) Comparative metabolomics reveals the metabolic variations between two endangered Taxus species (T. fuana and T. yunnanensis) in the Himalayas. BMC Plant Biol 18:197

Zhang YK, Wang YJ, Gupta P, Chen ZS (2015) Multidrug resistance proteins (MRPs) and cancer therapy. AAPS J 17:802-812

Zhao G, Zhao W, Han L, Ding J, Chang Y (2020) Metabolomics analysis of sea cucumber (Apostichopus japonicus) in different geographical origins using UPLC-Q-TOF/MS. Food Chem $333: 127453$

Zheng H, Zhang D, Zhao X (2019) Metabonomic study on the effect of transcriptional factor SINAC4 on tomato fruit metabolites. Food Sci 40:36-42

Zininga T, Ramatsui L, Shonhai A (2018) Heat shock proteins as immunomodulants. Molecules 23:2846

Publisher's Note Springer Nature remains neutral with regard to jurisdictional claims in published maps and institutional affiliations. 